\title{
Metodología para la identificación y priorización de las actividades económicas que contribuyen a la estrategia de innovación para la especialización inteligente: Caso región de Arequipa
}

Methodology for the identification and prioritization of economic activities that contribute to the innovation strategy for smart specialization: Case of the Arequipa Region

Meza Mauricio y Delgado Max

Universidad Católica de Santa María, Arequipa, Perú.

\section{INFORMACIÓN}

Historia del Artículo

Recepción: 13/05/2109

Revisión: 07/06/2019

Aceptación: 22/08/2019

\section{Palabras Clave}

Priorización de actividades, metodología de priorización, criterios de valorización

\section{Key Words}

Prioritization, methodology, innovation, system, qualitative, actors, specialization, intelli-gent.

\section{DOI}

https://doi.org/10.35286/veritas. v21i1.254

\section{RESUMEN}

El estudio presenta una propuesta metodológica para la identificación y priorización de actividades económicas que contribuyen a la estrategia de innovación para la especialización inteligente en la región de Arequipa, considera datos tanto cuantitativos como cualitativos, basados en información estadística y calificaciones otorgadas por los actores que representan la hélice cuádruple ( academia, gobierno, empresa y sociedad civil), del Sistema Regional de Innovación (SRI), respectivamente. Estos datos definidos en un marco de factores críticos, como el potencial de crecimiento económico, competitividad, conectividad y agrupación, permitirán determinar la priorización de las actividades económicas en la región. El resultado de la aplicación de la metodología prioriza la actividad minera; actividad agrícola-agroindustrial; y actividad manufacturera: textil de camélidos sudamericanos en la región de Arequipa, Perú.

\begin{abstract}
The study presents a methodological proposal for the identification and prioritization of economic activities that contribute to the innovation strategy for smart specialization in the Arequipa region, considers both quantitative and qualitative data, based on statistical information and qualifications granted by the actors representing the quadruple propeller (academy, government, business and civil society), of the Regional Innovation System (SRI), respectively. These data defined in a framework of critical factors, such as the potential for economic growth, competitiveness, connectivity and cluster, will allow determining the prioritization of economic activities in the region. The result of the application of the methodology prioritizes the mining activity; agriculture-agribusiness activity; and manufacturing activity: textile of South American camelids in the region of Arequipa, Peru.
\end{abstract}

\section{INTRODUCCIÓN}

Se desarrolla un método procedimental para la identificación de las actividades prioritarias para el desarrollo del ecosistema innovador de la región Arequipa, y contribuye para el fortalecimiento de políticas públicas. Se desarrolla la siguiente propuesta en una circunstancia en que los gobiernos regionales como los señala Storper (1997) han iniciado alianzas con las universidades y centros de investigación para la generación y/o transferencia de tecnologías, en aspectos que afianzan las relaciones regionales y locales entre estos actores sobre la cual yacen los sistemas regionales de innovación.

Se tienen experiencias relevantes en políticas públicas para el crecimiento focalizadas en la inversión vinculada al conocimiento y ventajas competitivas en el Gobierno de Canarias, mediante las expresadas en la Guía para la elaboración de estrategias de innovación para la especialización inteligente RIS3, A nivel latinoamericano resaltan las experiencias de México, Colombia y Chile. Con todo, la determinación de las áreas y la priorización de las actividades productivas que más contribuyen al desarrollo de una región parte de identificar las actividades importantes sobre ellas se dinamizan las políticas para desarrollar la innovación y la tecnología.

Se ha podido observar que en América Latina el proceso de planteamientos para el desarrollo en innovación y en tecnología carece de herramientas metodológicas y aportes teóricos para la priorización de sectores mediante combinaciones cualitativas y cuantitativas. En ese sentido, el presente trabajo sugiere una aproximación metodológica, la cual ha sido utilizada en el caso de la región Arequipa Perú. Considerando que varios estudios encuentran a la innovación como herramienta dinamizadora, esta podría tener efectos adversos (Hiernaux-Nicolás, 1999) cuando no se reconoce la importancia del análisis social en sus procesos (Klein y Fontan, 2004; Hillier, Moulaert y Nussbaumer, 2004). 


\section{Marco Teórico}

Para la identificación y priorización de los sectores productivos que contribuyen a la innovación en Arequipa 2017, de acuerdo a la guía RIS3, la priorización debe conducir a una transformación económica basada en cuatro principios generales:

- La relevancia de masa crítica de la región.

- La ventaja competitiva de una región basada en el conocimiento y/o ventajas naturales.

- La conectividad y la asociatividad empresarial (cadenas productivas y/o clústeres).

- El liderazgo colaborativo (sistemas regionales de innovación dinámicos).

La combinación del análisis cualitativo y cuantitativo con la técnica para Ordenar las preferencias mediante la Similitud a una Solución Ideal, o TOPSIS es usado junto a la jerarquía analítica propuesta por Saaty 1980, como parte de un conjunto de métodos permite considerar las apreciaciones subjetivas en juicios de sobre la importancia relativa de cada objetivo y que especifique una preferencia sobre el objetivo y permite la resolución de problemas multicriterio, multientorno y multiactores.

Identificando los criterios a evaluar entre diferentes alternativas, se debe construir una matriz cuadrada con los mismos criterios en filas y columnas, la matriz pareada se complica si por ejemplo se consideran $10+10$ sectores productivos, que requerirían 100 evaluaciones por cada especialista, lo cual complica el análisis, en este aspecto el presente estudio ha realizado una innovación también para la matriz de comparación pareada normalizada.

Analizando el modelo AHP, uno muy similar es el Analytic network process (ANP), este método subdivide al problema de análisis en objetivo, criterio y alternativas. La diferencia gravita en que se permiten relaciones de dependencia entre elementos de distintas ramas, mientras que en el AHP estas solo pueden ser unidireccionales.

Siguiendo con la rama de métodos ideados a ayudar a las personas que emiten juicios de calor, encontramos el método Macbeth, el cual es un método interactivo que tiene por finalidad cuantificar el atractivo que presenta cierto estímulo, al momento de emitir algún juicio (Bana e Costa, 2000). En términos generales, valorar información de tipo ordinal sobre las predilecciones de un directivo respecto de un conjunto de alternativas, no presenta mayor dificultad. Lastimosamente, en la mayor parte de las aplicaciones prácticas esta información no es suficientemente completa, para asegurar que los resultados sean significativos, pues a menudo es necesario saber, no solo que $\mathrm{x}$ es más atractivo que y, sino también cuánto más atractivo es. Muy poca gente posee información importante a priori (directamente de su mente al papel).

El juicio de expertos sobre un determinado tema puede llegar a ser considerado como subjetivo, pero no arbitrario por la experiencia. En este contexto, el método Delphi suele ser preferido debido a que utiliza el análisis prospectivo basado en la consulta a expertos (Okoli y Pawlowski, 2004; Camisón y Cruz, 2008), gozando de una gran aplicación en el área de las ciencias sociales (Corbetta, 2003, Landeta, 2006). Cabe considerar que este método reduce la posibilidad de sesgo en las opiniones, puesto que se pide, se recopila, se evalúa y se tabula opiniones independientes sin discusiones presenciales de grupo.

El Método Delphi, permite la estructuración de un proceso de comunicación grupal lo cual es efectivo a la hora de permitir a un grupo de individuos, como un todo, tratar un problema complejo (Linstone y Turoff, 1975). Así también permite llegar a un consenso grupal, lo cual posibilita la generación de conocimiento (Rowe y Wright, 1999). Aunque será posible encontrarse con situaciones en donde el consenso no se logra.

Pese a las ventajas del método, se muestran también inconvenientes, siendo uno de ellos, que los resultados de las opiniones de expertos podrían considerarse puntos de vista personales sin análisis de contexto, las opiniones podrían dar mayor peso a eventos pasados recientes, y no a lo que está ocurriendo en el momento o pueda ocurrir en el futuro. Por ello diversos autores consideran que sus resultados deben ser interpretados con precaución (Gupta y Clarke, 1996; Rowe, Wright y Bolger, 1991). También el método es muy sensible a la forma como se plantean las preguntas (ambigüedad, imprecisión) y hay que definir los estándares para el cuestionario, por ejemplo, las escalas a utilizar y, también, tener cuidado con la claridad de las preguntas. Dada la complementariedad de la información cuantitativa y cualitativa se necesita una metodología que incluya los datos cuantitativos y cualitativos; y que permita evaluar varias alternativas en un marco que incluya varios objetivos.

\section{METODOLOGÍA}

La metodología presenta tres fases, la primera dirigida a la identificación, selección y priorización de los factores críticos a considerar para la priorización de las actividades económicas, la segunda dirigida a la identificación de las actividades económicas a considerar y a la identificación y selección de los actores participantes, y la tercera encaminada a la priorización de las actividades económicas.

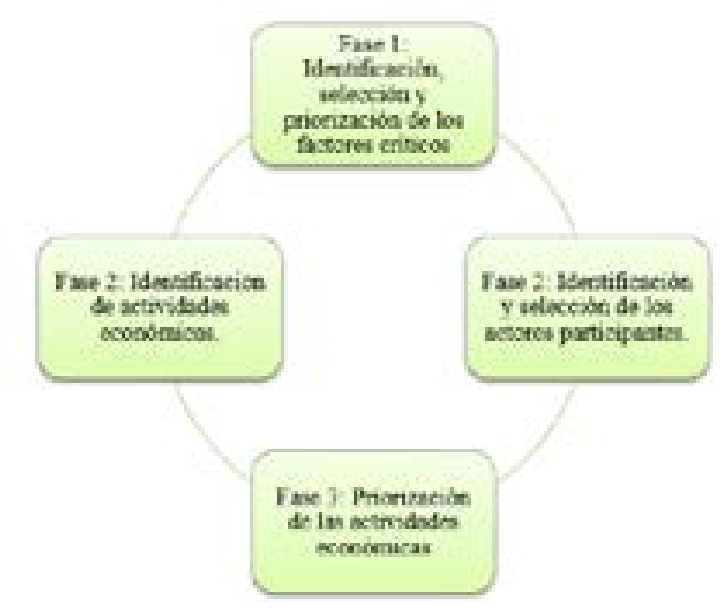

Fig. 1: Fases metodológicas. Fuente: Elaboración propia 
Fase 1: Identificación, selección y priorización de los factores críticos a considerar

Para la priorización se parte de la identificación de los factores críticos a considerar mediante una herramienta para la selección de criterios en medio de varias opciones. Cabe mencionar los aportes teóricos de la selección de proyectos, Corvellec and Macheridis 2010 considera la responsabilidad moral de esta compleja selección, y sugiere considerar la coproducción de la organización y el equipo selector de proyecto.

Siguiendo al Proceso de Análisis Jerárquico (Método AHP), desarrollado por Thomas L. Saaty que sugiere abordar un problema complejo de criterio múltiple mediante la jerarquización de los problemas planteados, el proceso se inicia con la determinación de los factores críticos considerando revisión bibliográfica de fuentes secundarias, el análisis de datos estadísticos y los instrumentos de planificación regional que evidencien de manera objetiva las contribuciones de las actividades productivas a la región, complementado con el principio de que la experiencia y el conocimiento de los actores son tan importantes como los datos utilizados en el proceso. Para el caso de la región Arequipa surgen para la innovación de especialización inteligente en una región, los siguientes criterios:

El primero es el factor crecimiento económico, medido por el Valor Agregado Bruto (VAB) de la producción, es decir se considera a las actividades que contribuyen en mayor medida al PBI regional, identificando como criterio complementario el nivel actual y potencial de las exportaciones, y la inversión planificada para las actividades económicas de la economía de la región; En esta parte se trabaja con datos estadísticos oficiales tomando los valores mayores por actividad; lo que determina las actividades prioritarias con el factor potencial de crecimiento económico

El segundo factor es la competitividad, en coherencia con Morales (2003) al hablar de competitividad sistémica para la implementación de políticas públicas en el nivel de competitividad meso son los que se relacionan con el capital intelectual, logístico y organizacional. entendida por el potencial que tiene la región en relación a sus ventajas comparativas y dotación de recursos naturales estratégicos que generen valor agregado a futuro, evidenciando el esfuerzo en investigación y desarrollo y el potencial de generación de conocimientos en cada actividad económica de la región.

El tercer factor es la conectividad de las actividades en su cadena productiva y en específico en los canales de distribución que movilizan y generan transacciones de bienes, servicios, información y personas entre distintos puntos del territorio regional, nacional e internacional, evidenciado en las cadenas logísticas de distribución, evidenciado como aporte a la competitividad del nivel regional o meso por Morales (2003). Se toma en cuenta las vías de comunicación accesibilidad a los puertos; al respecto un estudio del INEI tiene mapeado la conectividad por actividades económicas, las que se ha tomado para la ponderación

El cuarto factor toma en cuenta el potencial de generación de valor a partir de la potencialidad y pre existencia de los clústeres, que articulen varios sectores. Para el caso Boisier (1999) le llama capital sinérgico podrían tratarse de redes de cooperación para el desarrollo de proyectos comunes ó complementarios.

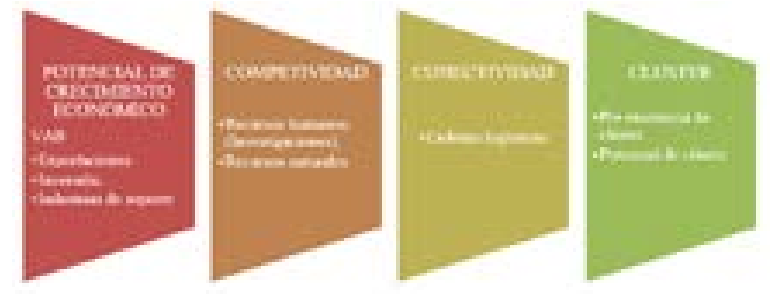

Fig. 2: Factores críticos e indicadores. Fuente: Elaboración propia

Fase 2: Identificación de los actores y las actividades económicas a evaluar con los factores críticos priorizados

Es de especial importancia el análisis de los actores en el ecosistema innovador considerando los siguientes criterios para su selección:

- El conocimiento, capital intelectual y de experiencia que puedan tener los actores en relación al ecosistema innovador que es considerado por Daniels and Noordhuis (2005) como un activo intangible. Este criterio también es valorado por Medaglia et al. (2008) como elemento del análisis de proyectos públicos atribuyéndole la utilidad como elemento para seleccionar de un banco de proyectos existentes.

- La participación de los representantes de las actividades económicas a analizar procedentes del sector público, representantes de la Academia, representantes de empresas y representantes de la sociedad civil. Esta selección se da porque la participación de los sectores puede ser considerado como una herramienta de sustento a las políticas públicas incluso Amin y Thrift (1993) definieron a las políticas públicas como formas de coordinación entre el sector público y privado.

Para el análisis y selección de las actividades económicas se considera los considerados en las estadísticas del estado con los siguientes criterios:

- A nivel territorial se aprecian varias actividades económicas en vigencia dando dinamismo al territorio.

- Las actividades económicas de acuerdo a la clasificación que realiza el INEI, sobre los datos de producción y aporte al PBI de las actividades económicas de una determinada región.

- Se evita considerar aquellas actividades relacionadas a organismos autónomos como por ejemplo la administración pública o las actividades relacionadas a servicios financieros, debido a que son actividades que dependen de la política monetaria y fiscal; y no presentan características diferenciadoras a nivel global.

Una limitación del estudio es que no considera los espacios emergentes que podrían pasar desapercibidos por la posibilidad de imitar comportamientos del contexto Mendez (2016). 


\section{Fase 3: Priorización}

Saaty en el AHP hace una jerarquización con prioridades que muestran la preferencia global para cada una de las alternativas de decisión, donde "trata de desmenuzar un problema y luego unir todas las soluciones de los subproblemas en una conclusión". Pese a las preeminencias del modelo, se requiere hacer comparaciones por parejas entre todas las alternativas, valorando el nivel de preferencia con una escala definida en el intervalo que va desde 1 (equivalencia), hasta 9 (preferencia extrema).

Considerando que en relación al AHP Jensen (1984), respecto a la escala utilizada sugiere que la escala no debería estar acotada al intervalo que va desde (1/9) hasta 9. Otro aspecto en contra es que su aplicación resulta excesivamente laboriosa, por la necesidad de comparar todas las parejas posibles de elementos. Al respecto, tanto en Takeda (1987), como en Harker (1987), se realizan propuestas tendientes a reducir la cantidad de comparaciones.

Es aquí donde se innova metodológicamente y se considera lo siguiente:

- Se establecen prioridades con una matriz de comparaciones global que considera las actividades económicas de la región, de acuerdo a cada factor crítico (potencial de crecimiento económico, competividad, conectividad y clúster), considerando datos cuantitativos y cualitativos.

- Los datos cuantitativos, basados en información estadística, considerados para el factor potencial de crecimiento económico y competividad, pertenecen a los resultados publicados por el Instituto Nacional de Estadística e Informática (INEI), el Banco Central de Reserva del Perú (BCRP); el Plan de Desarrollo Concertado Arequipa 2013 - 2021; y CONCYTEC; los datos estadísticos para el factor conectividad han sido extraídos del portal del Ministerio de Transportes y Comunicaciones (Cadenas Logísticas, 2015); y los datos para el factor clúster han tomado en consideración el documento "Mapeo de Clúster en el Perú" (Metis Gaia, 2013); para el periodo 2010- 2015.

- Los datos cualitativos corresponden a las calificaciones emitidas por el grupo de especialistas invitados, considerando la escala del 0 al 10 como ponderador de calificación menor al $0 \mathrm{y}$ de mayor al 10 .

Para la priorización se realizan los siguientes pasos:

Paso 1: Construcción de la matriz de datos para la priorización

En la tabla 1, podemos observar en la primera columna la descripción de las actividades económicas; y en la columna 2; observamos los factores críticos que priorizaran las actividades económicas.
Tabla 1: Matriz de datos para la priorización de las actividades económicas. Elaboración: Agenda Innovación Arequipa

\begin{tabular}{|c|c|c|c|c|}
\hline \multirow[b]{2}{*}{ ACTIVIDADES ECONOMICAS } & \multicolumn{4}{|c|}{$\begin{array}{l}\text { FACTORES } \\
\text { CRITI-COS }\end{array}$} \\
\hline & 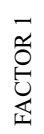 & 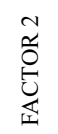 & 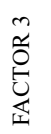 & 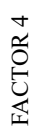 \\
\hline \multicolumn{5}{|l|}{ Agricultura, ganadería, caza silvicultura } \\
\hline \multicolumn{5}{|l|}{ Actividad 2} \\
\hline \multicolumn{5}{|l|}{ Actividad 3} \\
\hline \multicolumn{5}{|l|}{ Actividad 4} \\
\hline \multicolumn{5}{|l|}{ Actividad 5} \\
\hline
\end{tabular}

Paso 2.: Cálculo de ponderadores cuantitativos $y$ cualitativos

Para el cálculo de la ponderación cuantitativa se considera información estadística y para calcular la ponderación cualitativa se considera la calificación emitida por los actores representativos de la cuádruple hélice (academia, gobierno, empresa y sociedad civil), de la región.

A partir de la información estadística y las calificaciones expresadas por los actores invitados, se calcula la ponderación cuantitativa y cualitativa, correspondiente a cada actividad económica de acuerdo a cada factor crítico.

La estimación del promedio de la ponderación cuantitativa y cualitativa en base a los datos estadísticos y en base a las calificaciones otorgadas por los actores se explica con el siguiente ejemplo, en la tabla 2. En la primera columna, se expresan las actividades económicas de la región; en la columna $2 ; 3 ; 4 ;$ y 5 ; se expresaran los datos correspondientes a información estadística o las calificaciones recogidas; estos datos se deben sumar verticalmente, en el ejemplo la sumatoria de los datos del factor 1 es de 21 respectivamente. En las siguientes columnas $6 ; 7 ; 8$; y 9; se muestran los resultados de las contribuciones de cada actividad por cada factor crítico, se han calculado de la siguiente manera: se ha dividido el dato estadístico del factor 1 correspondiente a la actividad agricultura, ganadería, caza silvicultura, entre el total del factor $5 \div 21$; dando como resultado 0.24 en la columna 6 ; los resultados de la columna $6 ; 7 ; 8 ;$ y 9 ; deben sumar 1 .

Por ultimo en la columna 10; se halla el promedio de la ponderación cuantitativa o cualitativa de las columnas $6 ; 7 ; 8$; y 9 ; en el ejemplo, el promedio para la actividad agricultura, ganadería, caza silvicultura, da como resultado 0.22. Los datos de esta columna también deben sumar 1 . 
VÉRITAS Vol. 21 N¹ (2020) 25-34

Tabla 2: Ejemplo de estimación del promedio de la ponderación cuantitativa y cualitativa. Elaboración: Agenda Innovación Arequipa

\begin{tabular}{|c|c|c|c|c|c|c|c|c|c|}
\hline \multirow[b]{2}{*}{$\begin{array}{c}\text { ACTIVIDADES } \\
\text { ECONOMICAS DE LA } \\
\text { REGION AREQUIPA }\end{array}$} & \multicolumn{4}{|c|}{ DATOS } & \multicolumn{4}{|c|}{ PONDERACIÓN } & \multirow[b]{2}{*}{$\begin{array}{c}\text { PROMEDIO } \\
\text { PONDERACIÓN }\end{array}$} \\
\hline & 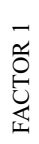 & 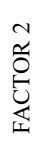 & 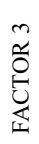 & 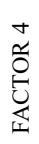 & 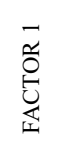 & 光 & 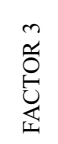 & 站 & \\
\hline $\begin{array}{l}\text { Agricultura, ganadería, } \\
\text { caza silvicultura }\end{array}$ & 5 & 5 & 7 & 1 & 0.24 & 0.28 & 0.28 & 0.08 & 0.22 \\
\hline Actividad 2 & 2 & 2 & 2 & 0 & 0.10 & 0.11 & 0.08 & 0.00 & 0.07 \\
\hline Actividad 3 & 7 & 5 & 7 & 7 & 0.33 & 0.28 & 0.28 & 0.54 & 0.36 \\
\hline Actividad 4 & 5 & 5 & 7 & 5 & 0.24 & 0.28 & 0.28 & 0.38 & 0.30 \\
\hline Actividad 5 & 2 & 1 & 2 & 0 & 0.10 & 0.06 & 0.08 & 0.00 & 0.06 \\
\hline TOTAL & 21 & 18 & 25 & 13 & 1 & 1 & 1 & 1 & 1 \\
\hline
\end{tabular}

\section{Paso 3: Normalización de las calificaciones cualitativas}

En la normalización, se trata de dar consistencia a las evaluaciones, la metodología AHP, desarrollada por Thomas L. Saaty considera, un índice de consistencia menor al 10\% para los juicios emitidos por el grupo de trabajo, en una matriz pareada. En la metodología propuesta al no trabajar con una matriz pareada, no se aplica el índice de consistencia, debido a que su aplicación resulta excesivamente laboriosa, Takeda (1987), Harker (1987) y considerando que la mente humana no opera al modo AHP, sino que realiza siempre comparaciones y ordenamientos globales, según Watson y Freeling (1982); es que, en la metodología propuesta, las calificaciones no se realizan mediante la comparación por pares, se realiza comparando todas las actividades económicas de acuerdo a cada factor crítico. Para disminuir la subjetividad en las calificaciones, primero, se entregara a cada participante la información estadística que le permita otorgar una calificación objetiva. Y segundo, se normaliza las calificaciones cualitativas como se explica a continuación.
En la tabla 3, se expone un ejemplo de estimación de la priorizacion de actividades económicas en la región. En la columna 1 se describen las actividades económicas; en la columna 2, se exponen los resultados de la ponderación cuantitativa; en la columna 3 , se observa los resultados de la ponderación cualitativa. Una vez que se cuente con la ponderación cuantitativa y cualitativa, se procede a normalizar, la ponderación cualitativa, con el fin de evitar posibles sesgos. La normalización de estas calificaciones resulta de multiplicar los resultados de la ponderación cuantitativa por los resultados de la ponderación cualitativa, como se observa en la columna 4 de la tabla 3 . Por ejemplo para la actividad 1 se multiplica la ponderación cuantitativa de la columna dos $(0.23)$; por la ponderación cualitativa de la columna tres ( 0.22$)$; dando como resultado la normalización de la ponderación cualitativa para la actividad 1, en la columna cuatro (0.05). Por ultimo en la columna 5 , se expone los resultados de la priorización:

Tabla 3: Ejemplo de estimación de la priorización en la región Arequipa. Elaboración: Agenda Innovación Arequipa

\begin{tabular}{ccccc}
\hline $\begin{array}{c}\text { ACTIVIDADES } \\
\text { ECONOMICAS DE LA } \\
\text { REGION AREQUIPA }\end{array}$ & $\begin{array}{c}\text { PONDERACION } \\
\text { CUANTITATIVA CON }\end{array}$ & $\begin{array}{c}\text { PONDERACION ESTADISTICA } \\
\text { BA }\end{array}$ & $\begin{array}{c}\text { CULITATIVA EN BASE A } \\
\text { CALIFICACIONES } \\
(\mathrm{b})\end{array}$ & $\begin{array}{c}\text { NORMALIZACION DE } \\
\text { LA PONDERACION } \\
\text { CUALITATIVA } \\
(\mathrm{a} * \mathrm{~b}=\mathrm{c})\end{array}$ \\
\hline Actividad 1 & 0.23 & 0.22 & 0.05 & 0.28 \\
Actividad 2 & 0.11 & 0.07 & 0.01 & 0.12 \\
Actividad 3 & 0.25 & 0.36 & 0.09 & 0.34 \\
Actividad 4 & 0.19 & 0.3 & 0.06 & 0.25 \\
Actividad 5 & 0.22 & 0.06 & 0.01 & 0.23 \\
\hline
\end{tabular}




\section{Paso 4: Resultados de la Priorización}

Para determinar la priorización, se retoma la tabla anterior, en la que, se muestra en la columna 5 los resultados de la priorización de actividades económicas como resultado de la suma de los datos de la ponderación cuantitativa (columna 2), con los datos de la ponderación cualitativa normalizada, (columna 4). Por ejemplo para la actividad 1 se suma el dato de la ponderación cuantitativa $(0.23)$ con el dato de la ponderación cualitativa normalizada (0.05); dando como resultado 0.28 en la columna de priorización.

La columna 5 determina un ranking de puntaje por cada actividad económica; este puntaje nos va permitir identificar la priorización; por ejemplo si debemos escoger tres actividades prioritarias, estas serían la actividad 3 ; 1 ; y 4 respectivamente.

\section{RESULTADOS}

La metodología sugerida ha sido aplicada e implementada en un análisis piloto para el caso de la Agenda de Innovación de Arequipa, mediante el proceso de la aplicación metodológica para la priorización de actividades económicas de la región como se observa en la figura 3.

A continuación la aplicación de la propuesta metodológica:

En la fase 1 de la metodología se ha identificado a los factores críticos que determinaran la priorización de las actividades económicas de la región: potencial de crecimiento económico (indicadores: VAB, exportaciones, inversión e industrias de soporte); competividad (indicadores: recursos humanos y naturales); conectividad (cadenas logísticas); y clúster (indicadores: preexistencia y potencial de clúster).

En la fase 2. Identificación de los actores y las actividades económicas a evaluar con los factores críticos priorizados, que colaboraron fueron:

a. Las instituciones del sector público fueron: Gobierno

Regional de Arequipa GRA; Centros de Innovación

Productiva y Transferencia Tecnológica (CITE)

Agroindustrial; CITE Textil Camélidos; CITE Cuero y Calzado; CITE Arequipa.

b. Las instituciones de la academia fueron: Universidad Nacional de San Agustín (UNSA); Universidad Católica de Santa María (UCSM); Universidad Católica San Pablo (UCSP); Universidad Alas Peruanas; Universidad La Salle; Universidad Tecnológica del Perú (UTP); TECSUP; SENATI; Instituto de Investigación y Desarrollo del Sur (IIDS); Instituto de Innovación Agraria (INIA); Instituto Agrario del Sur - IAS; Instituto Superior Tecnológico Público La Joya - IEST LA JOYA.

c. Las empresas fueron: Cámara de comercio e industria Arequipa, empresas agrícolas agroindustriales, empresas metalmecánicas, empresas industria textil.

d. Las instituciones de la sociedad civil fueron: Organizaciones No Gubernamentales (ONG) Desco, ONG Ceder, ONG El Taller.

En cuanto a las actividades económicas, se consideró la clasificación del Instituto Nacional de Estadística e Informática (INEI), como podemos observar en la tabla 4, las cuales son: agricultura, ganadería, caza y silvicultura; pesca y acuicultura; extracción de petróleo, gas, minerales y servicios conexos; manufactura; electricidad, gas y agua; construcción, comercio, mantenimiento y reparación de vehículos automotores y motocicletas; transporte, almacenamiento, correo y mensajería; turismo: alojamiento y restaurantes; telecomunicaciones y otros servicios de información.

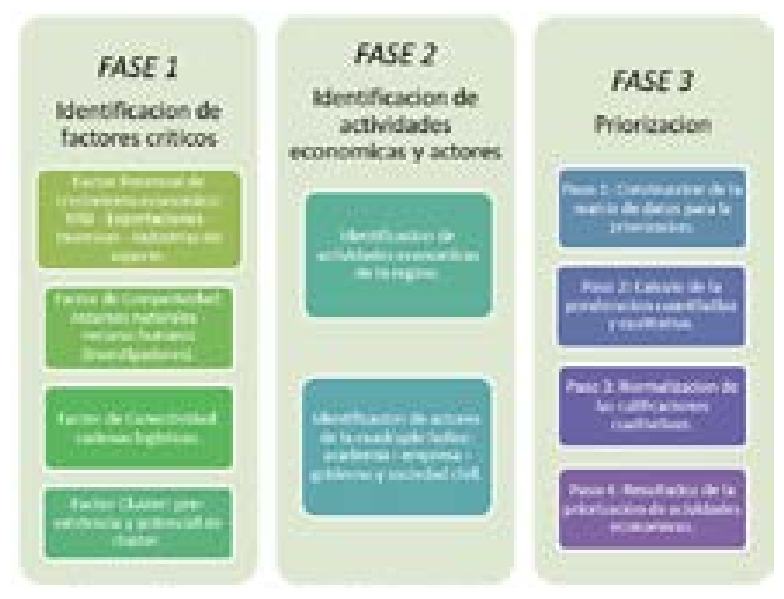

Fig. 3: Fases del proceso metodológico de priorización en la región Arequipa. Elaboración: Agenda Innovación Arequipa

Teniendo en cuenta los factores críticos y las actividades económicas de la región Arequipa, se procederá, en primer lugar, a la recolección y al tratamiento de los datos estadísticos para determinar la ponderación cuantitativa. En segundo lugar se procederá a la recolección y tratamiento de las calificaciones otorgadas por los actores, que determinaran la ponderación cualitativa. Para el caso de la ponderación cuantitativa, vamos a explicar cómo se realizara la recolección y tratamiento de los datos estadísticos con el factor crítico potencial de crecimiento económico, el cual considera los siguientes indicadores: valor agregado bruto; exportaciones; inversión del gobierno regional; e industria de soporte. Este procedimiento, se replicara en los demás indicadores de cada factor crítico.

\section{Fase 3: Priorización}

Para la priorización se realizan los siguientes pasos.

Paso 1: Construcción de la matriz de datos para la priorización

Considerando a Takeda (1987), Harker (1987) y Watson y Freeling (1982), la construcción de la matriz de datos va a tomar en cuenta las diez actividades económicas identificadas para la región Arequipa y los 4 factores críticos obteniendo 40 datos por cada actor invitado. No se consideran matrices pareadas debido a que la mente humana realiza siempre comparaciones y ordenamientos globales y resultaría muy laboriosa la obtención de 400 datos por cada actor invitado, datos correspondientes a la comparación pareada de cada actividad económica, por cada factor crítico.

\section{Paso 2: Cálculo de ponderadores cuantitativos y} cualitativos

Recordemos que se deben calcular tanto la ponderación cuantitativa en base a datos estadísticos y la ponderación cualitativa en base a las calificaciones otorgadas por los actores invitados. 


\section{a) Ponderación cuantitativa}

A continuación, en la siguiente tabla se muestra el detalle de los indicadores que se han tomado en cuenta para medir los factores críticos de la región Arequipa. Cada indicador (VAB, exportaciones, inversión, industrias de soporte, recursos naturales, grado de conocimiento, cadenas logísticas, preclúster y potencial de clúster), han obtenido una valoración de acuerdo a la información estadística publicada por instituciones de prestigio mencionadas en la metodología. La ponderación cuantitativa total resulta del promedio de ponderaciones de cada actividad económica de acuerdo a los indicadores que forman los factores críticos (potencial de crecimiento económico, competividad, conectividad, y clúster). La ponderación cuantitativa total determina la siguiente priorización de actividades económicas: minería, manufactura y agricultura, ganadería, caza y silvicultura.

En la columna 1 de la tabla 4; se observa que en la región Arequipa, la actividad que más contribuye al Valor Agregado Bruto es la Minería, seguida de la actividad manufactura, comercio, construcción, agricultura, transporte, telecomunicaciones, turismo y por último la actividad pesca.

En la columna 2, se observa que, las actividades que concentran las exportaciones son: la agricultura ganadería y silvicultura, minería, pesca, textil, minería no tradicional (siderometalúrgico, químico, metal mecánico, mineral no metálico). Como se puede observar, las exportaciones están lideradas por la minería en 1er lugar, el 2do lugar en exportaciones lo ocupa la actividad textil, en 3er lugar las industrias de soporte generadas por la minería (sidrero metalúrgico, químico, metal, mineral no metálico), y en 3er lugar la actividad agropecuaria y pesca.

En la columna 3, se observa que, las actividades relevantes identificados por el gobierno regional de Arequipa, en cuanto al presupuesto estimado, son: minería, agroindustrial, manufactura, turismo y pesca.

En la columna 4, son relevantes las industrias de soporte como es el caso de las industrias de metal mecánica, químicos, sidero metalúrgico, minerales no metálicos que aparecieron gracias a la existencia la actividad minera. Y la industria textil que se generó a consecuencia de la actividad agropecuaria, y por la demanda de pelos finos de alpaca del exterior. Estas industrias de soporte le generan un gran valor a la región por medio de sus exportaciones, ya que como hemos observado líneas atrás, forman parte de las exportaciones no tradicionales y generan valor a la actividad manufactura y minería.

Tabla 4: Detalle de la ponderación cuantitativa. Elaboración: Agenda Innovación Arequipa

\begin{tabular}{|c|c|c|c|c|c|c|c|c|c|c|}
\hline \multirow[b]{2}{*}{$\begin{array}{l}\text { ACTIVIDADES ECONOMICAS } \\
\text { DE LA REGION AREQUIPA }\end{array}$} & \multicolumn{4}{|c|}{$\begin{array}{l}\text { POTENCIAL DE CRECIMIENTO } \\
\text { ECONOMICO }\end{array}$} & \multicolumn{2}{|c|}{ COMPETTIVIDAD } & \multirow{2}{*}{$\begin{array}{l}\text { CONECTIVIDAD } \\
\end{array}$} & \multicolumn{2}{|c|}{ CLUSTER } & \multirow[b]{2}{*}{$\begin{array}{l}\text { PONDERACION } \\
\text { CUANTITATIVA } \\
\text { TOTAL }\end{array}$} \\
\hline & $\stackrel{m}{\lessgtr}$ & 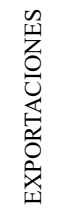 & 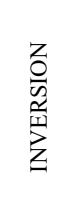 & 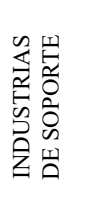 & 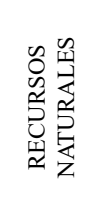 & 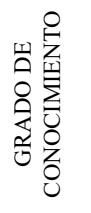 & & 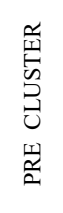 & 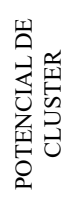 & \\
\hline $\begin{array}{c}\text { Agricultura, Ganadería, Caza y } \\
\text { Silvicultura }\end{array}$ & 0.09 & 0.03 & 0.007 & 0.00 & 0.33 & 0.16 & 0.40 & 0.00 & 0 & 0.11 \\
\hline Pesca y Acuicultura & 0.00 & 0.03 & 0.001 & 0.00 & 0.167 & 0.03 & 0.03 & 0.00 & 0 & 0.03 \\
\hline $\begin{array}{l}\text { Extracción de Petróleo, Gas, } \\
\text { Minerales y servicios conexos }\end{array}$ & 0.32 & 0.84 & 0.980 & 0.40 & 0.33 & 0.05 & 0.08 & 1 & 0.5 & 0.50 \\
\hline Manufactura & 0.20 & 0.10 & 0.009 & 0.60 & 0.00 & 0.42 & 0.50 & 0.00 & 0.5 & 0.26 \\
\hline Electricidad, Gas y Agua & 0.01 & 0.00 & 0.000 & 0.00 & 0.00 & 0.03 & 0.00 & 0.00 & 0.00 & 0.01 \\
\hline Construcción & 0.10 & 0.00 & 0.000 & 0.00 & 0.00 & 0.05 & 0.00 & 0.00 & 0.00 & 0.02 \\
\hline $\begin{array}{l}\text { Comercio, Mantenmiento } \\
\text { y Reparación de Vehículos } \\
\text { Automotores y Motoccletas }\end{array}$ & 0.14 & 0.00 & 0.000 & 0.00 & 0.00 & 0.00 & 0.00 & 0.00 & 0.00 & 0.02 \\
\hline $\begin{array}{l}\text { Transporte, Almaceamiento, } \\
\text { Correo y Mensajería }\end{array}$ & 0.07 & 0.00 & 0.003 & 0.00 & 0.00 & 0.00 & 0.00 & 0.00 & 0.00 & 0.01 \\
\hline $\begin{array}{l}\text { Turismo: Alojamiento y } \\
\text { Restaurantes }\end{array}$ & 0.03 & 0.00 & 0.000 & 0.00 & 0.17 & 0.00 & 0.00 & 0.00 & 0.00 & 0.02 \\
\hline $\begin{array}{l}\text { Telecomunicaciones y Otros } \\
\text { Servicios de Información }\end{array}$ & 0.04 & 0.00 & 0.000 & 0.00 & 0.00 & 0.25 & 0.00 & 0.00 & 0.00 & 0.03 \\
\hline TOTAL & 1 & 1 & 1 & 1 & 1 & 1 & 1 & 1 & 1 & 1 \\
\hline
\end{tabular}


En la columna 5, se observa que en cuanto a recursos naturales, las actividades predominantes son la de agricultura, ganadería, caza y silvicultura; y la de extracción de petróleo, gas, minerales y servicios conexos; seguidas de las actividades turismo: alojamiento y restaurantes; y pesca y acuicultura.

En la columna 6, en cuanto a los recursos humanos, el indicador grado de conocimiento revela que la ejecución de proyectos de acuerdo a la base de datos DINA de CONCYTEC, están concentrados en la actividad manufactura en 1er lugar, seguida de telecomunicaciones y otros servicios de información; agricultura, ganadería, caza y silvicultura; extracción de petróleo, gas, minerales y servicios conexos; construcción; y por ultimo las actividades de pesca y acuicultura; y electricidad, gas y agua.

En la columna 7, observamos que, las actividades económicas que hacen más uso de las redes de distribución terrestre, fluvial y aérea para trasladar productos tanto a nivel nacional como internacional, son las actividades de manufactura en ler lugar seguida de agricultura, ganadería, caza y silvicultura que ocupa el 2do lugar. Lo anterior nos sugiere que si la infraestructura de estas redes de conexión no mejora, podrían limitar el desarrollo económico de estas actividades. Siguiendo con el ranking de conectividad, el 3er lugar lo ocupa la actividad extracción de petróleo, gas, minerales y servicios conexos, debido a que la producción minera está dirigida al mercado externo más no al nacional. El 4to lugar lo ocupa la actividad pesca y acuicultura, debido también que la producción de esta actividad en su mayoría está dirigida al mercado externo.

En la columna 8, la actividad extracción de petróleo, gas, minerales y servicios conexos, es la que le va a generar una mayor contribución a la región en cuanto a la pre existencia de clúster y el potencial de clúster, ya que basta que se formalice el clúster auxiliar minero Lima Arequipa, para que empiece a generar valor tanto a nivel regional como nacional. $Y$ en 2do y no menos importante la actividad de Manufactura, específicamente la actividad textil al dar inicio a la formación del clúster de pelos finos Arequipa, Puno y Cuzco.

Tabla 5. Detalle de la Ponderación Cualitativa. Elaboración: Agenda Innovación Arequipa

\begin{tabular}{|c|c|c|c|c|c|c|c|c|c|c|c|}
\hline \multirow[b]{2}{*}{$\begin{array}{c}\text { ACTIVIDADES } \\
\text { ECONOMICAS DE LA } \\
\text { REGION AREQUIPA }\end{array}$} & \multirow[b]{2}{*}{$\begin{array}{l}\text { PONDERACION } \\
\text { CUANTITATIVA }\end{array}$} & \multicolumn{5}{|c|}{ CALIFICACION CUALITATIVA } & \multicolumn{5}{|c|}{ PONDERACION DE LA CALIFICACION CUALITATIVA } \\
\hline & & 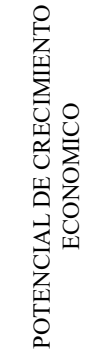 & 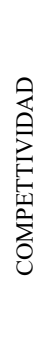 & 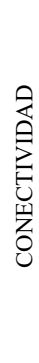 & 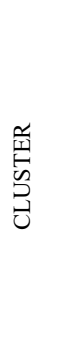 & 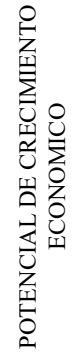 & 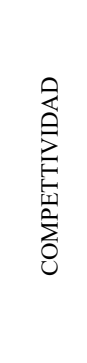 & 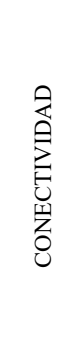 & 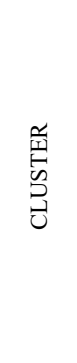 & $\begin{array}{c}\text { PONDERACION } \\
\text { CUALITATIVA }\end{array}$ & $\begin{array}{c}\text { NORMALIZACION } \\
\text { DE PONDERACION } \\
\text { CUALITATIVA }\end{array}$ \\
\hline $\begin{array}{l}\text { Agricultura, Ganadería, } \\
\text { Caza y Silvicultura }\end{array}$ & $11.3 \%$ & 5 & 4 & 5 & 3 & $9.7 \%$ & $10.3 \%$ & $9.8 \%$ & $9.4 \%$ & $9.8 \%$ & $1.1 \%$ \\
\hline Pesca y Acuicultura & $2.8 \%$ & 4 & 4 & 5 & 3 & $8.8 \%$ & $9.0 \%$ & $9.6 \%$ & $9.4 \%$ & $9.2 \%$ & $0.3 \%$ \\
\hline $\begin{array}{c}\text { Extracción de Petróleo, } \\
\text { Gas, Minerales y } \\
\text { servicios conexos }\end{array}$ & $49.9 \%$ & 5 & 5 & 5 & 4 & $10.1 \%$ & $11.0 \%$ & $10.6 \%$ & $10.3 \%$ & $10.5 \%$ & $5.2 \%$ \\
\hline Manufactura & $25.9 \%$ & 5 & 4 & 5 & 4 & $10.5 \%$ & $9.7 \%$ & $10.4 \%$ & $10.6 \%$ & $10.3 \%$ & $2.7 \%$ \\
\hline $\begin{array}{c}\text { Electricidad, Gas y } \\
\text { Agua }\end{array}$ & $0.5 \%$ & 5 & 5 & 5 & 4 & $10.5 \%$ & $10.5 \%$ & $10.0 \%$ & $10.3 \%$ & $10.3 \%$ & $0.1 \%$ \\
\hline Construcción & $1.7 \%$ & 6 & 4 & 5 & 3 & $11.1 \%$ & $9.2 \%$ & $10.2 \%$ & $9.7 \%$ & $10.0 \%$ & $0.2 \%$ \\
\hline $\begin{array}{c}\text { Comercio, } \\
\text { Mantenimiento } \\
\text { y Reparación de } \\
\text { Vehículos Automotores } \\
\text { y Motocicletas }\end{array}$ & $1.6 \%$ & 5 & 4 & 5 & 3 & $9.9 \%$ & $9.9 \%$ & $10.0 \%$ & $9.9 \%$ & $9.9 \%$ & $0.2 \%$ \\
\hline $\begin{array}{c}\text { Transporte, } \\
\text { Almacenamiento, } \\
\text { Correo y Mensajería }\end{array}$ & $0.8 \%$ & 5 & 4 & 5 & 3 & $9.3 \%$ & $10.1 \%$ & $9.8 \%$ & $9.9 \%$ & $9.8 \%$ & $0.1 \%$ \\
\hline $\begin{array}{l}\text { Turismo: Alojamiento y } \\
\text { Restaurantes }\end{array}$ & $2.2 \%$ & 5 & 5 & 5 & 4 & $10.5 \%$ & $10.5 \%$ & $10.4 \%$ & $10.8 \%$ & $10.6 \%$ & $0.2 \%$ \\
\hline $\begin{array}{l}\text { Telecomunicaciones } \\
\text { y Otros Servicios de } \\
\text { In-formación }\end{array}$ & $3.2 \%$ & 5 & 4 & 5 & 3 & $9.5 \%$ & $9.7 \%$ & $9.6 \%$ & $9.7 \%$ & $9.6 \%$ & $0.3 \%$ \\
\hline TOTAL & $100 \%$ & 51 & 43 & 52 & 35 & $100 \%$ & $100 \%$ & $100 \%$ & $100 \%$ & $100 \%$ & \\
\hline
\end{tabular}




\section{b) Ponderación cualitativa}

En la tabla 5 se detalla el cálculo de la ponderación cualitativa, basada en la recolección de las calificaciones otorgadas por los actores invitados, dichas calificaciones se recogieron en los talleres de sensibilización realizados. En tabla 5, en las columnas 3; 4; 5 y 6; se muestran las calificaciones otorgadas por los actores. En las columnas 7; $8 ; 9$ y 10 se ha realizado la ponderación de las calificaciones recogidas, el cálculo, por ejemplo, para la ponderación del factor potencial de crecimiento económico de la actividad económica agricultura, ha sido el siguiente: se ha dividido 5 : 51, (de la columna 2), que da como resultado $9.7 \%$ (columna 7), el mismo método se utiliza para calcular las demás ponderaciones cualitativas de las columnas $7 ; 8 ; 9$ y 10 . Posteriormente en la columna 11, se calcula el promedio de las ponderaciones cualitativas de cada actividad económica, cuya sumatoria da como resultado $100 \%$.

Paso 3. Normalización de las calificaciones cualitativas

Con respecto a las calificaciones emitidas por los actores, es importante disminuir el grado de subjetividad que se podría filtrar, en las calificaciones, como los puntos de vista personales, mayor importancia a eventos pasados, y no a lo que está ocurriendo en el momento o pueda ocurrir en el futuro, según Gupta y Clarke, 1996; Rowe, Wright y Bolger, 1991. Por lo que, en esta metodología se normalizaran los resultados de la priorización cualitativa, como se puede observar en la columna 3 de la tabla 6 . Retomando la tabla 5, el procedimiento es el siguiente: se realiza la normalización de la ponderación cualitativa, multiplicando la ponderación cuantitativa de la columna 2 por la ponderación cualitativa de la columna 11, por ejemplo, para la actividad agricultura, se multiplica el valor $11.3 \%$ (columna 2 ), por el valor $9.8 \%$ (columna 11), obteniendo como resultado el valor $1.1 \%$ (columna 12).

\section{Paso 4: Resultados de la priorización}

En la tabla 6, para calcular la priorización, se suman los valores de la ponderación cuantitativa (columna 2) y los valores resultantes de la normalización de la ponderación cualitativa (columna 3), por ejemplo, para la actividad agricultura, se suma el valor $11.3 \%$ más el valor $1.1 \%$, dando como resultado $12 \%$ (columna 4). La priorización cuantitativa representa el esfuerzo de instituciones como el BCRP; INEI y el GRA; y la priorización cualitativa representa la participación de actores de las cuatro hélices del Sistema Regional de Innovación de Arequipa (gobierno, academia, empresa y sociedad civil).
Tabla 6: Priorización de actividades económicas de la región Arequipa. Elaboración: Agenda Innovación Arequipa

\begin{tabular}{|c|c|c|c|}
\hline $\begin{array}{c}\text { ACTIVIDADES } \\
\text { ECONOMICAS DE LA } \\
\text { REGION AREQUIPA }\end{array}$ & $\begin{array}{l}\text { PONDERACION } \\
\text { CUANTITATIVA }\end{array}$ & $\begin{array}{c}\text { PONDERACION DE } \\
\text { LA CALIFICACION } \\
\text { CUALITATIVA } \\
\text { NORMALIZADA }\end{array}$ & PRIORIZACION \\
\hline $\begin{array}{c}\text { Agricultura, } \\
\text { Ganadería, Caza y } \\
\text { Silvicultura }\end{array}$ & $11.3 \%$ & $1.1 \%$ & $12 \%$ \\
\hline Pesca y Acuicultura & $2.8 \%$ & $0.3 \%$ & $3 \%$ \\
\hline $\begin{array}{c}\text { Extracción de } \\
\text { Petróleo, Gas, } \\
\text { Minerales y servicios } \\
\text { conexos }\end{array}$ & $49.9 \%$ & $5.2 \%$ & $55 \%$ \\
\hline Manufactura & $25.9 \%$ & $2.7 \%$ & $29 \%$ \\
\hline $\begin{array}{c}\text { Electricidad, Gas y } \\
\text { Agua }\end{array}$ & $0.5 \%$ & $0.1 \%$ & $1 \%$ \\
\hline Construcción & $1.7 \%$ & $0.2 \%$ & $2 \%$ \\
\hline $\begin{array}{c}\text { Comercio, } \\
\text { Mantenimiento } \\
\text { y Reparación } \\
\text { de Vehículos } \\
\text { Automotores y } \\
\text { Motocicletas }\end{array}$ & $1.6 \%$ & $0.2 \%$ & $2 \%$ \\
\hline $\begin{array}{c}\text { Transporte, } \\
\text { Almacenamiento, } \\
\text { Correo y Mensajería }\end{array}$ & $0.8 \%$ & $0.1 \%$ & $1 \%$ \\
\hline $\begin{array}{c}\text { Turismo: Alojamiento } \\
\text { y Restaurantes }\end{array}$ & $2.2 \%$ & $0.2 \%$ & $2 \%$ \\
\hline $\begin{array}{l}\text { Telecomunicaciones y } \\
\text { Otros } \\
\text { Servicios de Información }\end{array}$ & $3.2 \%$ & $0.3 \%$ & $4 \%$ \\
\hline
\end{tabular}

En base a la metodología propuesta las actividades económicas prioritarias de la región Arequipa, tomando en cuenta la ponderación cuantitativa y cualitativa son: la actividad minera, manufactura: textiles y actividad agrícola, agroindustria.

El desarrollo del sector minero y sus conexos en Arequipa es histórico y evidente al respecto Ramirez J, et al (2014) describe una vinculación entre el clúster minero y el sector metalmecánico. También ofrece potencial la manufactura de textiles donde resaltan los derivados de la fibra de camélidos, este hallazgo coincide con el presupuesto estimado de los proyectos y programas plasmados en el Plan de Desarrollo concertado de la Regional Arequipa 2013-2021.

Con la oportunidad de ampliación de la frontera agrícola mediante el proyecto de desarrollo agrícola Majes II y la incursión de tecnologías para la transformación de los productos agrícolas se puede afirmar que el desarrollo de la actividad agrícola - agroindustria está en crecimiento. 


\section{CONCLUSIÓN}

La priorización de las actividades económicas está en función a cuatro factores críticos, el primero es el potencial de crecimiento económico, medido por el valor agregado bruto, exportaciones, inversiones e industrias de soporte, el segundo es el de competividad, que resalta los recursos naturales y los recursos humanos (investigadores) de la región, el tercero es el de conectividad, medido por las cadenas logísticas de distribución de bienes y servicios tanto a nivel nacional como internacional y el cuarto es el de pre-existencia y potencial de clúster, a fin de conocer la capacidad de asociatividad en las actividades económicas de la región.

La propuesta metodológica de priorización de actividades económicas para una región, se basa en la recolección de datos cuantitativos basados en información estadística y datos cualitativos correspondientes a las calificaciones otorgadas por actores invitados pertenecientes a la cuádruple hélice (academia, gobierno, empresa y sociedad civil), del Sistema de Regional de Innovación (SRI). La priorización considera el resultado de datos cuantitativos y cualitativos normalizados, nos da como resultado un ranking con puntajes y en base a la metodología son: la actividad minera, manufactura: textiles y actividad agrícola, agroindustria.

\section{REFERENCIAS BIBLIOGRÁFICAS}

1. Amin, A.; Thrift, N. (1993). "Globalization, institutional thickness and local prospects". Revue d'Economie Regionale et Urbaine (Poitiers), 3, p. 405-427.

2. Aristóteles Yajure César (2015, Septiembre). Comparación de los métodos multicriterio AHP y AHP Difuso en la selección de la mejor tecnología para la producción de energía eléctrica a partir del carbón mineral. Sistema de Información Científica Red de Revistas Científicas de América Latina y el Caribe, España y Portugal, Scientia et Technica Año XX, Vol. 20, No. 3, de. Universidad Tecnológica de Pereira. ISSN 0122-1701. Obtenido el 24 de septiembre del 2017 en http://www.redalyc.org/html/849/84943818008/

3. Campos, Vanessa; Melián, Amparo; Navarro Joan; Sanchis Palacio, Ramón (2014, 2, April-June). El método Delphi como técnica de diagnóstico estratégico. Estudio empírico aplicado a las empresas de inserción en España. Sciencedirect, Volume 23, Pages 72-81. Obtenido el 24 de septiembre del 2017 en http://www.sciencedirect.com/science/article/pii/ S1019683813000620

4. Carreño Jaimes, Marisol (2009). El método Delphi: cuando dos cabezas piensan más que una en el desarrollo de guías de práctica clínica. Sciencedirect, vol. 38. Obtenido el 24 de septiembre del 2017 en http://www.scielo.org.co/pdf/rcp/v38n1/v38n1a13.pdf

5. Corvellec, Hervé; Nikos Macheridis (2010). The Moral Responsibility of Project Selectors. International Journal of Project Management p. 212-19. Obtenido el 15 de octubre del 2017 en http://www.sciencedirect. com/science/article/pii/S0263786309000611 (May 8, 2015).

6. Banae Costa, C.A.-Vansnick, J.C. (1994): “MACBETH - an interactive path towars the construction of cardinal value functions". Int. Trans. Oper. Res. 1, pp. 489-500.
7. Boisier Sergio. "El desarrollo territorial a partir de la construcción de capital sinergetico". Obtenido el 24 de septiembre del 2017 en http://www.cedet. edu.ar/Archivos/Bibliotecas_Archivos/capital\%20 sinerg\%C3\%A9tico95.pdf

8. Castellanos Castellanos, Dina Paola (2015). Selección de proyectos, un análisis del estado del arte a partir de artículos científicos. Obtenido el 24 de septiembre del 2017 en ttp://repositorio.uned.ac.cr/ reuned/bitstream/120809/824/1/Toma $\% 20 \mathrm{de} \% 20$ decisiones $\% 20$ con $\% 20$ criterios $\% 20$ multiples $\% 20$ un\%20resumen\%20conceptual.pdf

9. Ramirez J. et al (2014) Arequipa una estrategia para el crecimiento sostenido y la prosperidad

10. Gobierno Regional de Arequipa, (2015). Plan de Desarrollo Concertado. Obtenido el 4 de febrero del 2017 en http://www.regionarequipa.gob.pe/index.php/ planeamiento-y-organizacion/planes-de-desarrollo/ plan-desarrollo-concertado

11. Hennie, Daniels; Noordhuis, Henk (2005). "Project Selection Based on Intellectual Capital Scorecards." Intelligent Systems in Accounting, Finance and Management 13(1): 27-32. Obtenido el 24 de septiembre del 2017 en http://resolver.scholarsportal. info/resolve/1055615x/v13i0001/27_psboics

12. Hiernaux-Nicolás, D. (1999). Los senderos del cambio. Sociedad, tecnología y territorio en los albores del siglo XXI. México: Plaza Valdés.

13. Hillier, J., F. Moulaert y J. Nussbaumer (2004). "Trois essais sur le rôle de l'innovation sociale dans le développement territorial". Géographie, Économie, Société, 6, 2: 129-152.

14. Medaglia, Andrés L., Samuel B. Graves, and Jeffrey L. Ringuest. (2007). "A Multiobjective Evolutionary Approach for Linearly Constrained Project Selection under Uncertainty." European Journal of Operational Research 179(3): 869-94. Obtenido el 27 de septiembre del 2017 en http://www.sciencedirect.com/science/ article/pii/S0377221705007630

15. Osoriogómez, Juan Carlos; Orejuela Cabrera, Juan Pablo (2008, Septiembre). El proceso de análisis jerárquico (AHP) y la toma de decisiones multicriterio, ejemplo de aplicación. Scientia et Technica Año XIV, No 39, de. Universidad Tecnológica de Pereira. ISSN 0122-1701. Obtenido el 24 de septiembre del 2017 en file://D:/Documents/Downloads/3217-2381-1-PB.pdf

16. Velez-Pareja, Ignacio (2003, 29 Julio). The Delphi Method (El Metodo Delphi). Páginas 17. Obtenido el 24 de septiembre del 2017 en https://papers.ssrn.com/ sol3/papers.cfm?abstract_id $=420040$

17. Zanazzi, José Luis (2003, Agosto). Anomalías y supervivencia en el método de toma de decisiones de Saaty. Editorial Universitas, Córdoba, Volumen 1, paginas 148-170.

18. Obtenido el 24 de septiembre del 2017 en http:// www.efn.uncor.edu/departamentos/estruct/lgodoy/ Problemas\%20Conocimiento/10_Zanazzi.pdf 